\title{
Functional Specialization in the Human Medial Temporal Lobe
}

\author{
Morgan D. Barense, ${ }^{1}$ Timothy J. Bussey, ${ }^{2}$ Andy C. H. Lee, ${ }^{1}$ Timothy T. Rogers, ${ }^{1}$ R. Rhys Davies, ${ }^{3}$ Lisa M. Saksida, ${ }^{2}$ \\ Elisabeth A. Murray, ${ }^{4}$ and Kim S. Graham ${ }^{1}$ \\ ${ }^{1}$ Medical Research Council Cognition and Brain Sciences Unit, Cambridge CB2 2EF, United Kingdom, 2Department of Experimental Psychology, University \\ of Cambridge, Cambridge CB2 3EB, United Kingdom, ${ }^{3}$ Department of Clinical Neurosciences, University of Cambridge, Addenbrooke's Hospital, \\ Cambridge CB2 2QQ, United Kingdom, and ${ }^{4}$ Laboratory of Neuropsychology, National Institute of Mental Health, Bethesda, Maryland 20892
}

Investigations of memory in rats and nonhuman primates have demonstrated functional specialization within the medial temporal lobe (MTL), a set of heavily interconnected structures including the hippocampal formation and underlying entorhinal, perirhinal, and parahippocampal cortices. Most studies in humans, however, especially in patients with brain damage, suggest that the human MTL is a unitary memory system supporting all types of declarative memory, our conscious memory for facts and events. To resolve this discrepancy, amnesic patients with either selective hippocampal damage or more extensive MTL damage were tested on variations of an object discrimination task adapted from the nonhuman primate literature. Although both groups were equally impaired on standard recallbased memory tasks, they exhibited different profiles of performance on the object discrimination test, arguing against a unitary view of MTL function. Cases with selective hippocampal damage performed normally, whereas individuals with broader MTL lesions were impaired. Furthermore, deficits in this latter group were related not to the number of discriminations to be learned and remembered, but to the degree of "feature ambiguity," a property of visual discriminations that can emerge when features are part of both rewarded and unrewarded stimuli. These findings resolve contradictions between published studies in humans and animals and introduce a new way of characterizing the impairments that arise after damage to the MTL.

Key words: amnesia; hippocampus; medial temporal lobe; perirhinal cortex; object discrimination; declarative memory; perception; feature conjunctions

\section{Introduction}

Current theories of medial temporal lobe (MTL) memory organization are in conflict regarding two major issues. First, do regions in the MTL work in concert to support all forms of declarative memory (Squire et al., 2004), or can these structures be functionally dissociated, with the perirhinal cortex supporting recognition memory (Baxter and Murray, 2001; Mayes et al., 2004) and the hippocampus subserving recollection of contextual, episodic, or spatial information (Aggleton and Brown, 1999; Burgess et al., 2002; Winters et al., 2004)? Second, do these structures play an additional role in nonmnemonic perceptual processes, with the perirhinal cortex contributing to object perception (Buckley et al., 2001; Lee et al., 2005a) and the hippocampus contributing to spatial processing (Lee et al., 2005b)?

To date, much of the support for a functional division of labor in the MTL, both at a mnemonic and/or perceptual level, has

Received Feb. 11, 2005; revised Sept. 2, 2005; accepted Sept. 13, 2005.

This work was supported by the Medical Research Council, UK, and the Alzheimer's Research Trust, UK. R.R.D. is funded by the Wellcome Trust. We express our appreciation to all the participants in this study for their time and patience. We also thank Narinder Kapur and John Hodges for allowing us access to their patients, and Mark Baxter, Mark Buckley, David Gaffan, John Hodges, Rik Henson, Andrew Lawrence, Karalyn Patterson, and lan Nimmo-Smith for helpful feedback on this experiment.

Correspondence should be addressed to Morgan D. Barense, Medical Research Council Cognition and Brain Sciences Unit, 15 Chaucer Road, Cambridge CB2 2EF, UK. E-mail: morgan.barense@mrc-cbu.cam.ac.uk. DOI:10.1523/JNEUROSCI.2704-05.2005

Copyright $\odot 2005$ Society for Neuroscience $\quad$ 0270-6474/05/2510239-08\$15.00/0 come from studies in rats and nonhuman primates (Eacott et al., 1994; Murray and Mishkin, 1998; Buckley et al., 2001; Winters et al., 2004). Although there is some functional neuroimaging evidence for mnemonic specialization within the human MTL (Davachi and Wagner, 2002; Davachi et al., 2003; Giovanello et al., 2004; Ranganath et al., 2004), investigations in patients with brain injury typically suggest a single MTL memory system (Buffalo et al., 1998; Manns et al., 2003; Squire et al., 2004). Evidence in favor of a role for the MTL in perception is even scarcer. Unlike nonhuman primates with MTL damage (Eacott et al., 1994; Buckley et al., 2001; Bussey et al., 2002), amnesic patients often perform normally on simultaneous and short-delay, but not long-delay, object recognition (Buffalo et al., 1998; Holdstock et al., 2000; Stark and Squire, 2000).

One possible explanation for these contradictions is that human paradigms have failed to assess memory and perception by taxing the appropriate dimension highlighted in the animal literature. For example, monkeys with perirhinal lesions were impaired on concurrent object discriminations with a high, but not low, degree of "feature ambiguity," a property of visual discrimination problems that emerges when discriminating between complex objects with a large number of visual features in common (Bussey et al., 2002). These results suggest that rostral inferotemporal cortical regions, including perirhinal cortex, contain representations of complex conjunctions of stimulus 
features, whereas more caudal regions (e.g., V4, TEO) house the components from which these conjunctions are formed (Bussey and Saksida, 2002). If true, not all types of perceptual discrimination will reveal impairments in patients with MTL damage encompassing perirhinal cortex, only those taxing the ability to represent feature conjunctions.

To investigate this issue, the performance of two groups of amnesic patients, one with selective bilateral hippocampal damage and another with damage to MTL regions including perirhinal cortex, was assessed on concurrent object discriminations adapted from those used in monkeys. The test allowed us to ask two questions: (1) could we find evidence for dissociation of function in the MTL on object-based memory, and (2) would object feature ambiguity influence performance in individuals with MTL damage encompassing perirhinal cortex?

\section{Materials and Methods \\ Participants}

Eight amnesic patients with focal brain lesions participated in this study. Seven of these pa-

tients had structural magnetic resonance imaging (MRI) scans appropriate for visual rating (electronic versions suitable for volumetric analysis were not available). These were used to categorize the cases into the following two groups (Fig. 1, scan rating method): (1) those individuals with selective hippocampal involvement (hippocampal group; $n=5$ ) and (2) participants with broader medial temporal damage, including perirhinal cortex (MTL group; $n=3$ ). Of the three patients in the MTL group (age, 68.0 years; education, 10.3 years), two were viral encephalitis cases and the third had experienced traumatic intracerebral bleeding. Of the five patients in the hippocampal group (age, 48.7 years; education, 14.6 years), two had been diagnosed with viral encephalitis, one experienced cerebral anoxia in the context of suspected encephalitis, one had anoxia during status epilepticus, and one experienced carbon monoxideinduced hypoxia. One patient categorized in the hippocampal group refused to undergo additional scanning. We were unable to retrieve her previous scan, but the radiological report indicated selective hippocampal damage, and her performance on standard neuropsychological tests was indistinguishable from the other cases with selective hippocampal damage. Notably, exclusion of this patient did not significantly alter the experimental findings reported here.

For the experimental tests, 12 young (age, 52.5 years; education, 14.3 years) and 12 elderly (age, 66.1 years; education, 11.7 years) healthy subjects were age and education matched to the hippocampal group and the MTL cases (all $p>0.26$ ), respectively.

\section{Scan rating method}

To determine the degree of brain injury in the patients, two neurologists with extensive experience in methods for evaluating structural damage in neuropsychological patients used a visual rating scale modified from a previous study (Galton et al., 2001; Lee et al., 2005a). Hard copies of the coronal MRI scans of seven of the amnesic patients and 12 age-matched healthy controls (who did not participate in the experimental study) were assessed. All scans were presented randomly with identifying information obscured. The raters were blind to the aims of the investigation and identities of the individual participants.

The visual rating method has been validated successfully against volumetric measures (Galton et al., 2001) and emphasizes medial temporal areas, namely the entorhinal and perirhinal cortices. The rated regions and the method by which they were assessed is given below [from Lee et

al. (2005a)]. Other than the anterior hippocampus, which was assessed on a five-point scale (normal $=0$, severe atrophy $=4$ ) based on the work by Scheltens et al. (1992), all areas were rated using a four-point scale (normal $=0$, severe atrophy $=3$ ). Each measure showed satisfactory interrater reliability (average $\kappa$ value $=0.5)$ (Landis and Koch, 1977).

Anterior hippocampus. This was rated using the anterior-most pontine slice on a scale almost identical to that used by Galton et al. (2001) and Scheltens et al. (1992). The widths of the choroidal fissure and temporal horn of the lateral ventricle and the height of the hippocampal formation were assessed visually. This method is known to have good interrater and intrarater reliability and has been validated against both linear and volumetric measures obtained with different MRI sequences (Galton et al., 2001).

Anterior temporal pole. Assessment of this region was on a four-point scale and was based on the CSF space between the back of the orbit and the temporal pole.

Amygdala. This was rated on the scan slice anterior to the tip of the temporal horn, which corresponds to accepted MRI landmarks (Watson et al., 1997).

Lateral temporal lobe. Like the anterior hippocampus, this rating, which is most likely to correspond to area TE (Von Bonin and Bailey, 1947), was based on the slice through the anterior pons and depended on the cortical thickness of the superior and middle temporal gyri.

Posterior hippocampus. This was rated on the anterior-most slice through the cerebral aqueduct, in parallel with the anterior measure, according to the width of the temporal horn and the height of the hippocampal formation.

Medial temporal lobe. Cortical thickness was rated at the following four points: the midpoint of the crown of the parahippocampal gyrus (corresponding to entorhinal cortex), the midpoint of the medial bank of the collateral sulcus (corresponding to the boundary between entorhinal and perirhinal cortices), the midpoint of the lateral bank of the collateral sulcus (corresponding to perirhinal cortex), and the medial bank of the occipitotemporal sulcus (corresponding to the transition between perirhinal and isocortex) (Insausti et al., 1998). In each case, the ratings were performed on the slice showing the collateral sulcus at its longest. If there was significant anatomic distortion (e.g., that the gyral landmarks were unusable), then a severe atrophy rating was recorded.

On the basis of these ratings (Table 1), the patients with available scans 
Table 1. Structural MRI scan ratings (with SDs) for various brain regions (ordered from anterior to posterior location in the brain), averaged across both hemispheres

\begin{tabular}{|c|c|c|c|c|c|c|c|c|c|}
\hline & AntTemp & Amyg & PHG & MBCS & LBCS & MBOS & AntHC & LatTemp & PostHC \\
\hline $\mathrm{HC1}$ & $1^{*}$ & 1 & $0.75^{*}$ & 0.75 & 0.25 & 0 & $1.5^{*}$ & 1 & 0.75 \\
\hline $\mathrm{HC} 2$ & 0 & 0.5 & 0.25 & 0.5 & 0.25 & 0 & $2^{*}$ & 0 & 0.25 \\
\hline $\mathrm{HC} 3$ & 0 & 0 & $0.75^{*}$ & 0.75 & 0.5 & 0.25 & $1.25^{*}$ & 0.5 & 1 \\
\hline HC4 & 0.5 & 0.25 & 0 & 0.75 & 0.25 & 0.25 & $1.5^{*}$ & 0.25 & 1 \\
\hline MTL1 & $2^{*}$ & $2.25^{*}$ & $1.5^{*}$ & $1^{*}$ & $1.25^{*}$ & $2^{*}$ & $1.75^{*}$ & $1.75^{*}$ & $1.75^{*}$ \\
\hline MTL2 & $2^{*}$ & $3^{*}$ & $2.5^{*}$ & $2.75^{*}$ & $2.5^{*}$ & $2^{*}$ & $3^{*}$ & 1 & $2.75^{*}$ \\
\hline MTL3 & $1.75^{*}$ & $2.75^{*}$ & $2.75^{*}$ & $2.75^{*}$ & $2.5^{*}$ & $2.5^{*}$ & $2^{*}$ & 0.5 & $2^{*}$ \\
\hline HC group mean & $0.375(0.479)$ & $0.438(0.427)$ & $0.438(0.375)$ & $0.688(0.125)$ & $0.313(0.125)$ & $0.125(0.144)$ & $1.56^{*}(0.315)$ & $0.438(0.427)$ & $0.750(0.354)$ \\
\hline MTL group mean & $1.917^{*}(0.144)$ & $2.667^{*}(0.382)$ & $2.250^{*}(0.661)$ & $2.167^{*}(1.01)$ & $2.083^{*}(0.722)$ & $2.167^{*}(0.289)$ & $2.25^{*}(0.661)$ & $1.083(0.629)$ & $2.167^{*}(0.520)$ \\
\hline Control group mean & $0.313(0.284)$ & $0.375(0.483)$ & $0.188(0.188)$ & $0.521(0.291)$ & $0.271(0.310)$ & $0.333(0.289)$ & $0.458(0.382)$ & $0.458(0.411)$ & $0.271(0.361)$ \\
\hline
\end{tabular}

0 indicates no visible damage; 3 (4 for anterior hippocampus) indicates complete absence of area. The rating for each individual patient and the mean of each subject group is listed. HC, Hippocampal; AntTemp, anterior temporal cortex Amyg, amygdala; PHG, parahippocampal gyrus (corresponding to entorhinal cortex); MBCS, medial bank of collateral sulcus (corresponding to the transition between entorhinal and perirhinal cortex); LBCS, lateral bank of collateral sulcus (corresponding to perirhinal cortex); MBOS, medial bank of occipitotemporal sulcus (corresponding to the transition between perirhinal and isocortex); AntHC, anterior hippocampus; LatTemp, lateral temporal cortex (likely to correspond to TE); PostHC, posterior hippocampus.

*Significant difference compared with control mean.

were divided into those that had selective damage to the hippocampus bilaterally $(n=5)$ and those that had larger MTL lesions $(n=3)$, including damage to the perirhinal cortex, as well as some injury to anterior temporal lobe regions (Fig. 1). Because age was not a significant factor influencing the ratings obtained for healthy controls, the control subjects were considered as a single group. In addition, statistical analyses revealed no significant differences between the ratings obtained for left and right hemisphere regions (all $p>0.01$ ), and consequently, the ratings for each area were averaged across both hemispheres.

Table 1 displays the ratings for each individual patient and the mean scores for each of the three subject groups (hippocampal, MTL, and control). A repeated-measures ANOVA with a within-group factor of region and a between-group factor of subject group revealed a significant difference in scores across the nine brain areas rated $\left(F_{(8,128)}=5.64 ; p<\right.$ $0.001)$, a significant overall difference between the three subject groups $\left(F_{(2,16)}=80.15 ; p<0.001\right)$, and a significant region-by-subject group interaction $\left(F_{(16,128)}=4.17 ; p<0.001\right)$. One-way ANOVAs confirmed a significant group difference on all brain areas $\left(\right.$ all $\left.F_{(2,16)}>17 ; p<0.001\right)$ other than the lateral temporal lobe measure $\left(F_{(2,16)}=2.52 ; p>0.1\right)$. Post hoc analyses on the regions in which there was a significant group difference indicated significantly greater atrophy of the anterior hippocampus compared with the control group $(p<0.001)$ in the hippocampal group but no other significant differences (all $p>0.1$ ). In contrast, the MTL group received significantly greater rating scores compared with the control group on all measures (all $p<0.001$ ) for which the one-way ANOVAs revealed significant group differences.

Because damage lateral to the perirhinal cortex (i.e., area TE) would present a possible confound in the interpretation of our findings, it is reassuring that the MTL group, when compared with controls, did not show significant atrophy in our lateral temporal lobe region, with two of three cases possessing no significant damage to this area. Although it is currently unclear exactly what region in the human brain corresponds to area TE in the macaque brain (Von Bonin and Bailey, 1947; Seltzer and Pandya, 1978), area TE in macaques is known to occupy the inferior and middle temporal gyri, the latter of which was included in the lateral temporal lobe rating. Consistent with this, the profile of performance in the MTL group did not match existing knowledge of the effects of damage to area TE in nonhuman primates. Lee et al. (2005a,b) reported normal fine color discrimination in these three cases, a process thought to be dependent on lateral temporal areas in macaque monkeys (Horel, 1994; Buckley et al., 1997). Furthermore, the two cases that did not show significant lateral temporal lobe atrophy were impaired on the experimental tests reported below, indicating that the individual with more lateral damage did not drive the effects reported in the present study.

\section{Neuropsychological battery}

The patients were administered a series of standardized neuropsychological tests to assess their memory and visual perception. In summary, these demonstrated that both groups of patients had deficits in episodic memory and were equally impaired on recall-based memory tests. For in- stance, both patient groups performed poorly on measures of episodic recall, such as the logical memory (Wechsler Memory Scale-Revised, stories 1 and 2) immediate recall (hippocampal, 23.6\%; MTL, 19.5\%), delayed recall (hippocampal, 5.1\%; MTL, 2.8\%), and on delayed recall of the Rey complex figure (hippocampal, 16.4\%; MTL, 10.6\%). Independent samples $t$ tests revealed no significant differences between the two groups on these tests $(p=0.67,0.71,0.66$, respectively). In contrast, there was a significant difference between the two groups on the Warrington recognition memory test for faces $(p<0.01)$, with normal performance in the hippocampal group (91.2\%) but a significant deficit in the MTL group (62.7\%). Visuoperceptual performance as measured by the Benton face test (hippocampal, 89.6\%; MTL, 79.0\%), Rey complex figure copy (hippocampal, 99.4\%; MTL, 92.1\%), and visual object space perception battery (both groups passed all object and space tests) was within the normal control range on all tasks, although comparisons between the two groups revealed a significant difference on the Benton face test $(p<0.01)$ but not on the Rey complex figure copy $(p=0.24)$. Whereas the hippocampal group exhibited intact semantic function, the MTL group was mildly impaired on semantic memory, including wordpicture matching (hippocampal, 99.7\%; MTL, 88.5\%; $p<0.05$ ), naming (hippocampal, 97.8\%; MTL, 67.2\%; $p<0.01$ ), and the pyramids and palm trees test (pictures) (hippocampal, 99.2\%; MTL, 89.7\%; $p=0.05$ ).

\section{Behavioral procedure}

Participants were presented with four types of object stimuli with varying levels of semantic familiarity ("blobs," "barcodes," "bugs," and "beasts"). Each stimulus item was the composite of two explicitly defined features, or components, of the stimuli (e.g., legs and body) (Fig. 2). Across each stimulus type, there were three levels of perceptual discrimination: (1) minimum feature ambiguity, in which no object features were ambiguous (i.e., both features were unique to target and nontarget objects); (2) intermediate feature ambiguity, in which one-half of the features were ambiguous (i.e., one feature appeared in both a target and nontarget, but the other feature was unique to the target); and (3) maximum feature ambiguity, in which all features were ambiguous (i.e., both features of the target also appear separately in nontargets). In the maximum condition, it was only the conjunction of the two features that correctly distinguished targets from nontargets (e.g., black body and yellow legs); the problem could not be solved on the basis of a single feature (e.g., black body), because there were no individual features that were unique to the targets. Critically, the number of objects to be remembered was held constant across the different conditions, but as feature ambiguity was increased, the demand on learning conjunctions of features was increased parametrically.

On each trial, two objects were presented $\sim 15 \mathrm{~cm}$ apart on a touchscreen, and one object was arbitrarily designated correct (target) and the other incorrect (nontarget). Touching either stimulus resulted in the offset of the stimulus display, accompanied by a pleasant, high-pitched tone if the target was chosen and an unpleasant, low-pitched tone if the nontarget was chosen. The position of the target on the right or the left of the screen was 
(a)
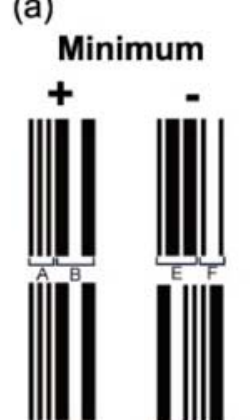

III

III
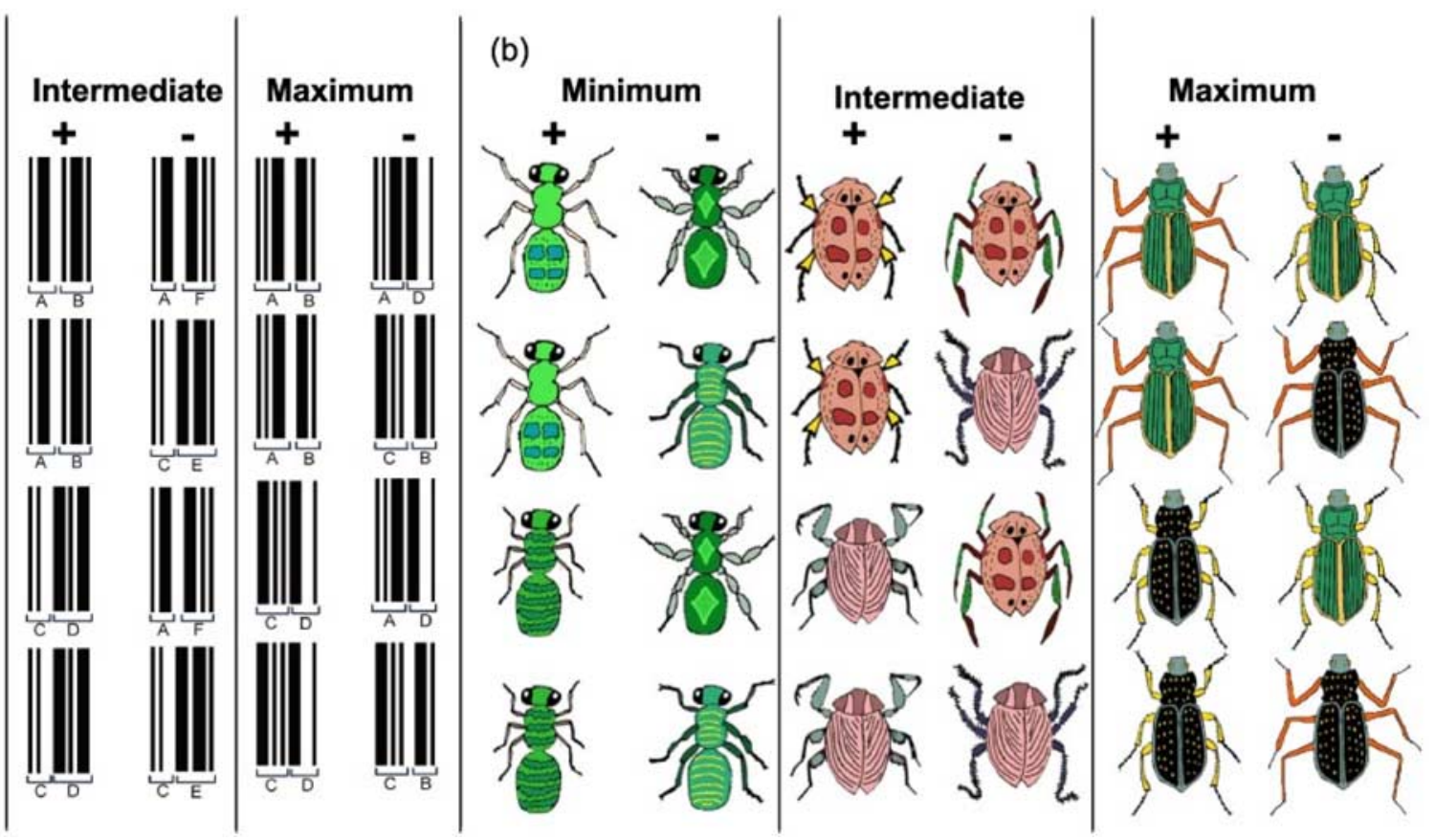

Figure 2. Concurrent object discrimination task. Subjects learned 11 discrimination problems, in which the number of objects was held constant, but the degree of feature ambiguity was varied systematically. Each discrimination problem consisted of four objects, presented in pairs. Two of the four objects were designated correct (targets), and two were designated incorrect (nontargets). In each pair, only one object was a target (shown here on the left). The pairs of objects were presented continuously in a pseudorandom order until the subject selected the target objects for eight consecutive trials. Stimuli, Each object consisted of the conjunction of the following two stimulus features: barcode components ( $\boldsymbol{a}$; individual features shown as letters for illustrative purposes) and bug parts ( $\boldsymbol{b}$; body and legs). Shape and fill were the manipulated features for the blobs stimulus set; body plan and coat pattern were used for the beast stimulus set. For examples of these stimuli, see Figure 3. Ambiguity, There were three feature ambiguity conditions: minimum ambiguity, in which no features were explicitly ambiguous (i.e., each feature was consistently either part of a target or a nontarget); intermediate ambiguity, in which one feature in each object (e.g., legs) was ambiguous; and maximum ambiguity, in which all features were ambiguous (i.e., each feature was simultaneously present in a target and nontarget object).

randomized. The testing was divided into rounds based on stimulus type, with the following 11 rounds in total: minimum-, intermediate-, and maximum-ambiguity blobs; minimum-, intermediate-, and maximumambiguity barcodes; minimum-, intermediate-, and maximum-ambiguity bugs; and minimum- and maximum-ambiguity beasts (Fig. 2). There was no intermediate condition for the beast stimulus set, because it was not possible to generate four living animals with components that conformed to the requirements of this condition. Each round consisted of four different pairs of four objects (a four-pair concurrent discrimination), meaning that each target would appear with each nontarget and vice versa. The pairs were presented in blocks of four, such that each pair appeared once and only once within the block; within each block, the order of pairs was randomized. When a criterion of eight consecutive correct trials was reached, the round would terminate, and after a short delay, the subject would begin another task condition. The order of presentation of the conditions was counterbalanced so that the maximum condition of each stimulus type appeared before the minimum condition of the same stimulus type for one-half of the subjects and vice versa for the remaining participants. The blob stimuli were administered on a separate occasion to both patients and controls. An average of 6 months separated the two testing sessions.

Informed consent was obtained from all subjects. This study received ethical approval from the Cambridge and Southampton Health Authority Local Research Ethics Committees (UK).

\section{Results}

The prevailing view of memory organization predicts that both groups of amnesic patients should show impairments on all conditions of this task, because it is a rapidly learned two-choice discrimination task. Rapid learning is thought to be a defining characteristic of declarative memory and would thus require an intact hippocampus (Teng et al., 2000; Zola and Squire, 2000). This view also makes no provision for impairments being influenced by feature ambiguity (Stark and Squire, 2000; Squire et al.,
2004). Instead, the degree of impairment should be modulated by the extent of damage within the MTL (hippocampal group less impaired than MTL cases) (Zola-Morgan et al., 1994). In contrast, theories guided by work in nonhuman primates predict (1) that there should be different profiles of performance between the hippocampal and MTL groups, with normal performance in the former (Baxter and Murray, 2001) and (2) that the MTL patients should be impaired when feature ambiguity is high but not when it is low (Buckley et al., 2001; Bussey et al., 2002). In addition, it is possible that familiarity might influence performance in the MTL cases, with greater deficits evident on novel stimuli (Lee et al., 2005b).

Statistical analyses on the acquisition data (shown as errors to criterion in Fig. 3) indicated that the two patient groups did not perform similarly across the different task conditions. The data for the four subject groups were subjected to four repeatedmeasures ANOVAs based on stimulus type (i.e., blobs, barcodes, bugs, and beasts). A single within-subject factor of ambiguity was incorporated with three levels (or two, in the case of the beast stimuli) corresponding to the degree of feature ambiguity (i.e., minimum, intermediate, and maximum). Given that the subject groups were structured (i.e., the young controls were chosen to match the hippocampal patients, and the elderly controls were selected to match the MTL patients), two between-subject factors, each with two levels, were included: (1) "health" with the levels patient (incorporating both patient groups) and control (incorporating both control groups) and (2) "lesion type" with the levels hippocampal (incorporating the hippocampal group and their matched controls) and MTL (incorporating the MTL group and their matched controls). The Huynh-Feldt correction 
(a)

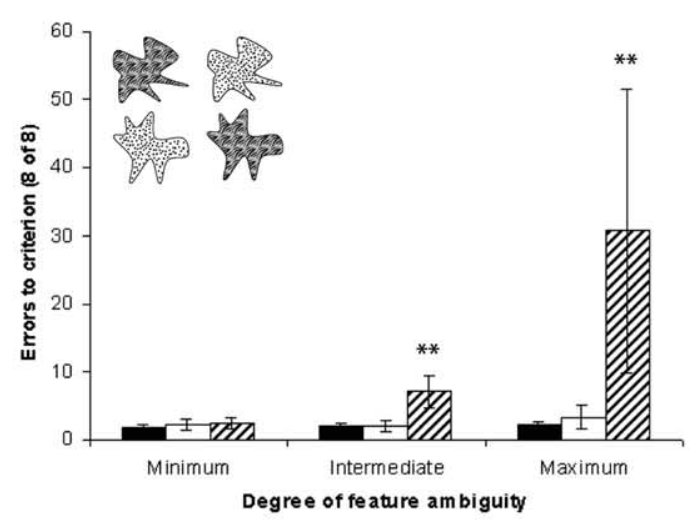

(b)

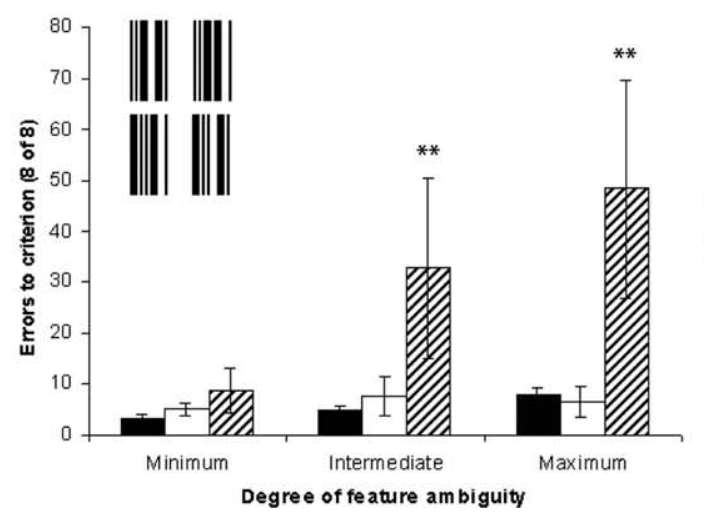

(c)
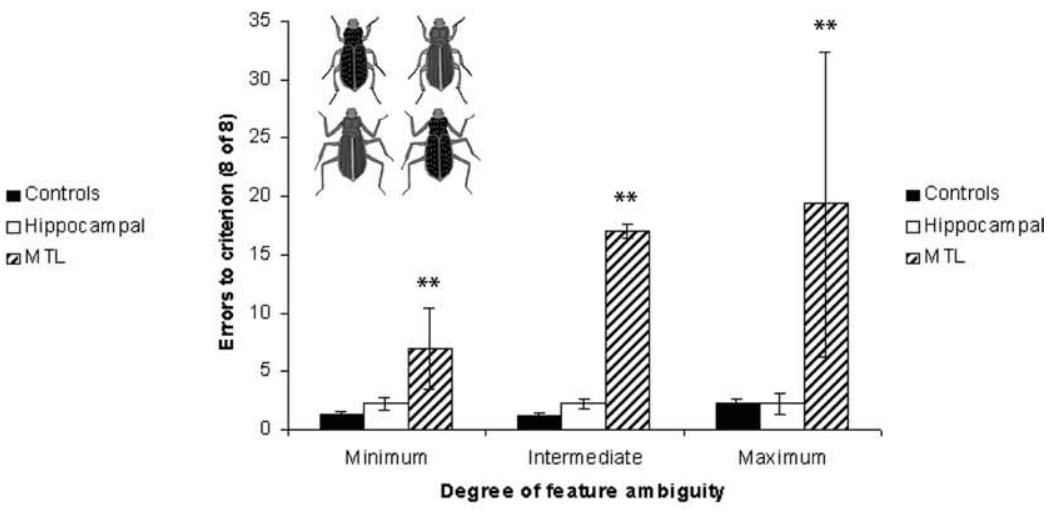

(d)

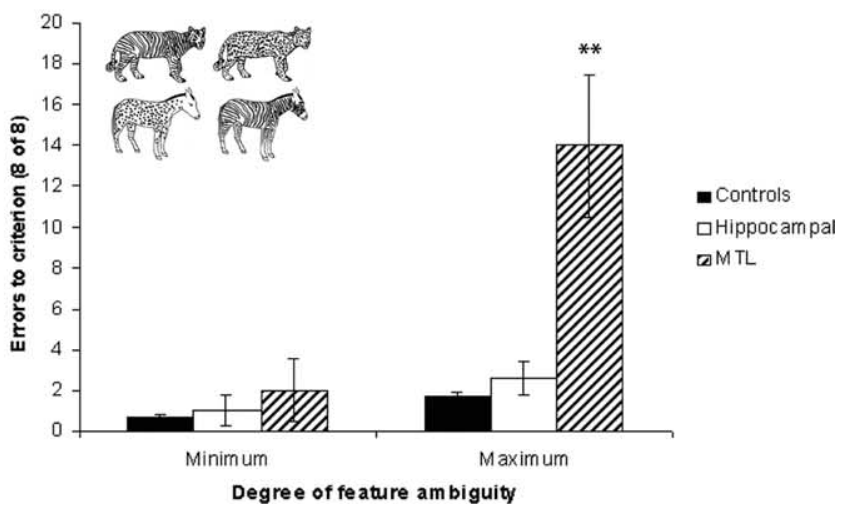

Figure 3. Mean errors to criterion (8 consecutive correct responses) for the two patient groups and two control groups (averaged) for blobs (a), barcodes $(\boldsymbol{b})$, bugs $(\boldsymbol{c})$, and beasts $(\boldsymbol{d})$ are shown. There was no significant difference between the young and old controls. One MTL patient was unable to achieve the criterion of eight consecutive correct for maximum-ambiguity blobs, intermediate-ambiguity barcodes, and maximum-ambiguity barcodes ( $>140$ trials). Objects used in the maximum-ambiguity condition of each stimulus type are shown on each graph. Correct objects are depicted on the left; incorrect objects are on the right. Error bars represent SEM. ${ }^{* *} p<0.01$ (MTL group vs control).

was used in cases in which Mauchley's $W$ statistic implied that sphericity could not be assumed. This analysis showed that the interaction between health and lesion type was significant for all stimulus types (all $p<0.001$ ), suggesting that the difference between the MTL group and their matched controls was significantly greater than that between the hippocampal patients and their respective controls on all stimuli.

In the ANOVA described above, the three-way interaction between health, lesion type, and ambiguity was also significant for all stimulus types (all $p<0.05$ ). This interaction revealed that the difference between the MTL group performance compared with the elderly control participants and the hippocampal group performance compared with the young control participants varied in magnitude as ambiguity increased. To investigate this further, the results from each individual condition (e.g., minimum blobs, intermediate blobs, maximum blobs, minimum barcodes, etc.) were analyzed separately using univariate ANOVAs. For each ANOVA, the same two between-subject factors of health and lesion type were included with a dependent variable of performance on each level of ambiguity. The interaction between health and lesion type was significant on all conditions in which the objects possessed ambiguous features (i.e., intermediate and maximum conditions of all stimulus types, all $p<0.01$ ) but not on the minimum-ambiguity conditions of blobs, barcodes, or beasts (all $p>0.40$ ). There was also a significant interaction between health and lesion type for the minimum-ambiguity condition of bugs $(p<0.01)$.

Within each of the tasks that showed an interaction between health and lesion type, we then performed $t$ tests to compare the performance of each patient group with their own control group. These revealed that on discriminations of objects with ambiguous features, the MTL patients performed significantly worse than their control group (all $p<0.01$ ). In contrast, the hippocampal group patients were not significantly different from their control group on these conditions (all $p$ between 0.12 and $0.96)$. These $t$ tests demonstrated that the significant interaction between health and lesion type in the discriminations with feature ambiguity arose because the MTL patients were impaired on these tasks, whereas the hippocampal patients performed within the normal range. $t$ tests to compare the performance of each patient group with its own control group on the minimumambiguity conditions revealed normal performance of the hippocampal group on all conditions (all $p>0.22$ ) and the MTL group for blobs, barcodes, and beasts (all $p>0.13$ ). The MTL group was impaired on the minimum-ambiguity condition of the bugs $(p<0.01)$, a result predominantly driven by the poor performance of one patient.

To rule out the possible explanation of task difficulty for the observed results in the MTL group, it is important to show that task difficulty does not increase with greater feature ambiguity. 
To address this issue, the data for the MTL control group were subjected to four repeated-measures ANOVAs based on stimulus type (i.e., blobs, barcodes, bugs, and beasts). A single withinsubject factor of ambiguity was incorporated with three levels (or two, in the case of the beast stimuli) corresponding to the degree of feature ambiguity (i.e., minimum, intermediate, and maximum). These analyses revealed no effect of ambiguity for any of the stimulus types (all $p$ between 0.08 and 0.4 ). Post hoc analyses (Bonferroni-corrected $t$ tests) were then performed on the performance data of the MTL control group across the different conditions within each stimulus type (e.g., minimum blobs vs intermediate blobs, intermediate blobs vs maximum blobs, minimum blobs vs maximum blobs, etc.). Of the 10 comparisons in total, none were significant (all adjusted $p$ between 1.0 and 0.08 ), indicating that this group of controls found all conditions similarly difficult.

One potential problem with this analysis, however, is that a sample size of 12 subjects may be too small to detect differences in task difficulty. To examine this possibility, we investigated the performance of a larger number of subjects $(n=$ 26; age, 59.5 years; education, 13.3 years) that were recruited to match three different patient groups [the MTL and the hippocampal group reported here and a group of patients with semantic dementia reported by Barense et al. (2005)]. The same 10 pairwise comparisons described above (Bonferronicorrected $t$ tests) were performed across the different conditions within each stimulus type. Only two of the comparisons (minimum barcodes vs maximum barcodes and minimum beasts vs maximum beasts) were significant $(p<0.01)$. For the large majority of the comparisons ( 8 of 10$)$, there was no difference in difficulty across conditions (all adjusted $p$ between 1 and 0.2). Thus, given that for the overwhelming majority of comparisons, there was no evidence for poorer performance in controls as feature ambiguity increased, a pattern not true of the MTL group, it seems extremely unlikely that differences in task difficulty can account for the observed pattern of results in the MTL patient group.

\section{Discussion}

The present study reveals striking convergence with results obtained in nonhuman primates (Bussey et al., 2002). Humans with MTL damage, including perirhinal cortex, were able to discriminate between objects with low, but not high, feature ambiguity. This pattern was evident for novel (blobs and barcodes) and familiar (bugs and beasts) objects, with more impairment (mean $z$-score $=-14.6$ ) on novel stimuli (maximum-ambiguity blobs) than on matched familiar objects with similar features (maximum-ambiguity beasts; mean $z$-score $=-9.5$ ). Strikingly, humans with selective hippocampal damage performed normally on all discriminations, regardless of feature ambiguity. This study, therefore, provides a parsimonious explanation for the discrepant results in the neuropsychological literature, suggesting clear specialization of function within the MTL and a modulatory effect of feature ambiguity in cases with perirhinal cortex damage.

The pattern of deficits in the MTL group cannot be attributed to task difficulty, because controls found the minimum, intermediate, and maximum conditions of each stimulus type equally difficult. Furthermore, the number of items to be discriminated was held constant across conditions. In fact, there were twice as many features in the minimum condition (eight) as in the maximum condition (four), indicating that the total number of presented features was inversely related to magnitude of impairment in the MTL group. Because controls learned all conditions equally quickly, the deficits reported here were not mediated by speed of learning. This finding runs counter to theories that consider rapid learning to be a defining characteristic of declarative memory and gradual, incremental learning to be supported by a nondeclarative procedural system independent of the MTL (Teng et al., 2000; Zola and Squire, 2000). The parametric manipulation of feature ambiguity between different conditions elicited both normal and impaired performance within the same MTL patients, thereby demonstrating that feature ambiguity (not learning speed, difficulty, or number of items to be discriminated) was the critical factor in determining the involvement of the cortex surrounding the hippocampus.

The MTL group did possess more extensive hippocampal damage than the hippocampal group. It is possible, therefore, that the poorer performance of the MTL group is attributable to this difference. Although the current study does not allow us to reject this possibility definitively, because only a single dissociation was demonstrated, there are several reasons why it is unlikely.

First, a view that holds that the degree of impairment should correlate with the amount of MTL damage would predict that patients with selective hippocampal damage should be at least mildly impaired on this declarative task, especially for very difficult discriminations (e.g., barcodes). There was no evidence for this here.

Second, the two patient groups were equally impaired on standard measures of recall. In fact, given the severe impairment in recall of both visual and verbal material in the hippocampal group, one cannot argue that the lesions in this group were functionally ineffective. Furthermore, all participants were unimpaired on the minimum-ambiguity discriminations, indicating that on at least some measures of memory function, the two patient groups were matched for performance. It was on discriminations between objects with a high degree of feature ambiguity, and in recognition memory for faces, which inherently possess a large number of overlapping features, that a selective impairment in the MTL group emerged.

Finally, powerful dissociations have now been documented on similar tasks requiring object or scene discrimination. Patients with semantic dementia, a condition associated with disproportionate atrophy to perirhinal cortex compared with other MTL structures (Davies et al., 2004), demonstrated a similar effect of feature ambiguity to that seen in the MTL group on the same tasks reported here (Barense et al., 2005). Furthermore, A. C. H. Lee, T. J. Bussey, E. A. Murray, N. Levi, L. M. Saksida, J. R. Hodges, and K. S. Graham (unpublished observations) found that patients with semantic dementia were impaired in the visual discrimination of faces but not scenes, whereas patients with Alzheimer's disease, who have less involvement of perirhinal cortex but severe hippocampal damage (Davies et al., 2004), showed particular difficulties with scene discrimination, with better face perception. These findings confirm that cases with involvement of perirhinal cortex show difficulties with object perception when the discriminations require conjunctions of object features (e.g., face discriminations), whereas cases with predominant hippocampal involvement are unimpaired on object tasks but perform poorly when spatial processing is stressed. Together, the results from these studies challenge alternative explanations for the data reported here, either in terms of "degree of hippocampal involvement" (i.e., greater hippocampal damage resulting in greater object discrimination deficits) and task difficulty (i.e., impairment only evident on hard discriminations). 
These results cannot be explained easily by prevailing views of the MTL. For example, a view positing a single declarative memory system within the MTL cannot account for the intact performance on all conditions and stimulus types in our hippocampal group. Such a theory predicts that damage to any component of the MTL memory system should impair performance on this declarative memory task. By showing that the cortex surrounding the hippocampus can function independently of a damaged hippocampus, at least in learning object discriminations, the present study implies specialization in the MTL. Furthermore, the intact performance of the MTL group on the minimum conditions reveals that the cortex surrounding the hippocampus, including perirhinal cortex, is only necessary for this form of declarative memory when the discriminations have a high degree of feature ambiguity.

The failure of previous studies to find perceptual deficits in humans with perirhinal damage (Buffalo et al., 1998; Holdstock et al., 2000; Stark and Squire, 2000) may reflect the use of objects with insufficient feature ambiguity, rather than a fundamental functional difference between the human and monkey MTL (Tyler et al., 2004; Lee et al., 2005a,b). This hypothesis, however, remains to be tested on paradigms used by other researchers. Thus, it would be useful to undertake a delayed-match-to-sample experiment incorporating a variable mnemonic demand but also manipulating feature ambiguity of the presented stimuli. This would provide a powerful test of whether feature ambiguity is the critical, and only, cause of the object memory impairments seen after broad MTL lesions and under what circumstances, if any, this factor interacts with delay.

One prominent theory holds that memory for relationships among perceptually distinct items (termed relational memory) is dependent on the hippocampus, whereas memory for individual objects is dependent on structures in the parahippocampal region (Eichenbaum et al., 1994; Cohen et al., 1997; Ryan et al., 2000; Eichenbaum and Cohen, 2001). This view postulates that parahippocampal structures house conjunctive representations of stimulus elements, thereby supporting memory for single objects. The present study is consistent with this view. Nonhippocampal MTL regions (i.e., perirhinal cortex) supported learning of configurations of features within individual objects independently of the hippocampus. The findings are also consistent with neuroimaging studies showing different patterns of activation across MTL regions, in particular those demonstrating perirhinal cortex activation for item-based memory of words and objects but not for their source or context (Davachi et al., 2003; Ranganath et al., 2004).

Because both patient groups possessed hippocampal damage, the present study cannot address directly functional specialization within the hippocampus itself. There is accruing evidence, however, that the hippocampus, and not its surrounding MTL regions, is critically involved in memory for associations between items and the context in which they are presented (Davachi and Wagner, 2002; Giovanello et al., 2004; Ranganath et al., 2004) and is especially important in computing the relationships between objects and features constituting a scene (Gaffan, 1994; King et al., 2004; Ryan and Cohen, 2004; Lee et al., 2005a,b). The role of contextual information was minimal in the present study, which may explain why patients with hippocampal damage were not impaired.

It is worth noting that the impairments observed in the MTL group do not necessarily reflect a purely mnemonic impairment per se, because they were capable of remembering the target cue if it consisted of unambiguous features. It was only when the target consisted of a conjunction of overlapping features that the MTL patients were impaired. This deficit could reflect a selective inability to remember complex stimuli with ambiguous features, but it might equally reflect an inability to perceive or create a representation of such stimuli at the time of encoding. Either explanation challenges long-standing conceptions of human amnesia, and additional research must determine whether the same neural mechanisms in MTL structures underlie both memory and perception. Furthermore, such studies must address how the deficits documented here may relate to mnemonic impairments that are not overtly perceptual or in the visual object domain (e.g., story recall or word recognition memory) but are so widely associated with the broader profile of human amnesia.

In conclusion, our findings are a significant first demonstration that the human MTL, like its nonhuman primate counterpart, is not exclusively specialized for all forms of object memory but is necessary for object discriminations only when the items to be remembered have a high degree of feature ambiguity. Furthermore, these abilities are supported by structures outside the hippocampus, indicating that the hippocampus is not critical for all forms of declarative memory.

\section{References}

Aggleton JP, Brown MW (1999) Episodic memory, amnesia, and the hippocampal-anterior thalamic axis. Behav Brain Sci 22:425-444; discussion 444-489.

Barense MD, Bussey TJ, Lee ACH, Rogers TT, Hodges JR, Saksida LM, Murray EA, Graham KS (2005) Feature ambiguity influences performance on object discriminations in patients with damage to perirhinal cortex. Paper presented at 12th Annual Meeting of the Cognitive Neuroscience Society, April.

Baxter MG, Murray EA (2001) Opposite relationship of hippocampal and rhinal cortex damage to delayed nonmatching-to-sample deficits in monkeys. Hippocampus 11:61-71.

Buckley MJ, Gaffan D, Murray EA (1997) Functional double dissociation between two inferior temporal cortical areas: perirhinal cortex versus middle temporal gyrus. J Neurophysiol 77:587-598.

Buckley MJ, Booth MC, Rolls ET, Gaffan D (2001) Selective perceptual impairments after perirhinal cortex ablation. J Neurosci 21:9824-9836.

Buffalo ED, Reber PJ, Squire LR (1998) The human perirhinal cortex and recognition memory. Hippocampus 8:330-339.

Burgess N, Maguire EA, O'Keefe J (2002) The human hippocampus and spatial and episodic memory. Neuron 35:625-641.

Bussey TJ, Saksida LM (2002) The organization of visual object representations: a connectionist model of effects of lesions in perirhinal cortex. Eur J Neurosci 15:355-364.

Bussey TJ, Saksida LM, Murray EA (2002) Perirhinal cortex resolves feature ambiguity in complex visual discriminations. Eur J Neurosci 15:365-374.

Cohen NJ, Poldrack RA, Eichenbaum H (1997) Memory for items and memory for relations in the procedural/declarative memory framework. Memory 5:131-178.

Davachi L, Wagner AD (2002) Hippocampal contributions to episodic encoding: insights from relational and item-based learning. J Neurophysiol 88:982-990.

Davachi L, Mitchell JP, Wagner AD (2003) Multiple routes to memory: distinct medial temporal lobe processes build item and source memories. Proc Natl Acad Sci USA 100:2157-2162.

Davies RR, Graham KS, Xuereb JH, Williams GB, Hodges JR (2004) The human perirhinal cortex and semantic memory. Eur J Neurosci 20:2441-2446.

Eacott MJ, Gaffan D, Murray EA (1994) Preserved recognition memory for small sets, and impaired stimulus identification for large sets, following rhinal cortex ablations in monkeys. Eur J Neurosci 6:1466-1478.

Eichenbaum H, Cohen NJ (2001) From conditioning to conscious recollection: memory systems of the brain. New York: Oxford UP.

Eichenbaum H, Otto T, Cohen NJ (1994) Two functional components of the hippocampal memory system. Behav Brain Sci 17:449-518.

Gaffan D (1994) Scene-specific memory for objects: a model of episodic 
memory impairment in monkeys with fornix transection. J Cogn Neurosci 6:305-320.

Galton CJ, Gomez-Anson B, Antoun N, Scheltens P, Patterson K, Graves M, Sahakian BJ, Hodges JR (2001) Temporal lobe rating scale: application to Alzheimer's disease and frontotemporal dementia. J Neurol Neurosurg Psychiatry 70:165-173.

Giovanello KS, Schnyer DM, Verfaellie M (2004) A critical role for the anterior hippocampus in relational memory: evidence from an fMRI study comparing associative and item recognition. Hippocampus 14:5-8.

Holdstock JS, Gutnikov SA, Gaffan D, Mayes AR (2000) Perceptual and mnemonic matching-to-sample in humans: contributions of the hippocampus, perirhinal and other medial temporal lobe cortices. Cortex 36:301-322.

Horel JA (1994) Retrieval of color and form during suppression of temporal cortex with cold. Behav Brain Res 65:165-172.

Insausti R, Juottonen K, Soininen H, Insausti AM, Partanen K, Vainio P, Laakso MP, Pitkanen A (1998) MR volumetric analysis of the human entorhinal, perirhinal, and temporopolar cortices. AJNR Am J Neuroradiol 19:659-671.

King JA, Trinkler I, Hartley T, Vargha-Khadem F, Burgess N (2004) The hippocampal role in spatial memory and the familiarity-recollection distinction: a case study. Neuropsychology 18:405-417.

Landis JR, Koch GG (1977) The measurement of observer agreement for categorical data. Biometrics 33:159-174.

Lee ACH, Bussey TJ, Murray EA, Saksida LM, Epstein RA, Kapur N, Hodges JR, Graham KS (2005a) Perceptual deficits in amnesia: challenging the medial temporal lobe "mnemonic" view. Neuropsychologia 43:1-11.

Lee ACH, Buckley MJ, Pegman SJ, Spiers H, Scahill VL, Gaffan D, Bussey TJ, Davies RR, Kapur N, Hodges JR, Graham KS (2005b) Specialization in the medial temporal lobe for processing of objects and scenes. Hippocampus 15:782-797.

Manns JR, Hopkins RO, Reed JM, Kitchener EG, Squire LR (2003) Recognition memory and the human hippocampus. Neuron 37:171-180.

Mayes AR, Holdstock JS, Isaac CL, Montaldi D, Grigor J, Gummer A, Cariga P, Downes JJ, Tsivilis D, Gaffan D, Gong Q, Norman KA (2004) Associative recognition in a patient with selective hippocampal lesions and relatively normal item recognition. Hippocampus 14:763-784.

Murray EA, Mishkin M (1998) Object recognition and location memory in monkeys with excitotoxic lesions of the amygdala and hippocampus. J Neurosci 18:6568-6582.

Ranganath C, Yonelinas AP, Cohen MX, Dy CJ, Tom SM, D’Esposito M
(2004) Dissociable correlates of recollection and familiarity within the medial temporal lobes. Neuropsychologia 42:2-13.

Ryan JD, Cohen NJ (2004) Processing and short-term retention of relational information in amnesia. Neuropsychologia 42:497-511.

Ryan JD, Althoff RR, Whitlow S, Cohen NJ (2000) Amnesia is a deficit in relational memory. Psychol Sci 11:454-461.

Scheltens P, Leys D, Barkhof F, Huglo D, Weinstein HC, Vermersch P, Kuiper M, Steinling M, Wolters EC, Valk J (1992) Atrophy of medial temporal lobes on MRI in "probable" Alzheimer's disease and normal ageing: diagnostic value and neuropsychological correlates. J Neurol Neurosurg Psychiatry 55:967-972.

Seltzer B, Pandya DN (1978) Afferent cortical connections and architectonics of the superior temporal sulcus and surrounding cortex in the rhesus monkey. Brain Res 149:1-24.

Squire LR, Stark CE, Clark RE (2004) The medial temporal lobe. Annu Rev Neurosci 27:279-306.

Stark CE, Squire LR (2000) Intact visual perceptual discrimination in humans in the absence of perirhinal cortex. Learn Mem 7:273-278.

Teng E, Stefanacci L, Squire LR, Zola SM (2000) Contrasting effects on discrimination learning after hippocampal lesions and conjoint hippocampal-caudate lesions in monkeys. J Neurosci 20:3853-3863.

Tyler LK, Stamatakis EA, Bright P, Acres K, Abdallah S, Rodd JM, Moss HE (2004) Processing objects at different levels of specificity. J Cogn Neurosci $16: 351-362$.

Von Bonin G, Bailey P (1947) The neocortex of Macaca mulatta. Urbana, IL: University of Illinois.

Watson C, Jack Jr CR, Cendes F (1997) Volumetric magnetic resonance imaging. Clinical applications and contributions to the understanding of temporal lobe epilepsy. Arch Neurol 54:1521-1531.

Winters BD, Forwood SE, Cowell RA, Saksida LM, Bussey TJ (2004) Double dissociation between the effects of peri-postrhinal cortex and hippocampal lesions on tests of object recognition and spatial memory: heterogeneity of function within the temporal lobe. J Neurosci 24:5901-5908.

Zola SM, Squire LR (2000) The medial temporal lobe and the hippocampus. In: The Oxford handbook of memory (Tulving E, Craik FIM, eds), pp 485-500. Oxford: Oxford UP.

Zola-Morgan S, Squire LR, Ramus SJ (1994) Severity of memory impairment in monkeys as a function of locus and extent of damage within the medial temporal lobe memory system. Hippocampus $4: 483-495$. 